\title{
List of interviews
}

\begin{tabular}{|c|c|c|c|}
\hline Institution & $\begin{array}{l}\text { Transcript } \\
\text { code }\end{array}$ & Date of interview & $\begin{array}{l}\text { Location of } \\
\text { interview }\end{array}$ \\
\hline EEAS, Brussels & PO1 & 20/08/2019 & Brussels \\
\hline EEAS, EU Delegation & $\mathrm{PO} 2$ & $20 / 08 / 2019$ & Phone interview \\
\hline EEAS, Brussels & PO3 & 04/09/2019 & Brussels \\
\hline EEAS, Brussels & PO4 & 05/09/2019 & Brussels \\
\hline EEAS, Brussels & PO5 & 09/09/2019 & Brussels \\
\hline EEAS, Brussels & PO6 & 09/09/2019 & Brussels \\
\hline EEAS, Brussels & PO7 & $11 / 09 / 2019$ & Brussels \\
\hline EEAS, Brussels & PO8 & 11/09/2019 & Brussels \\
\hline EEAS, Brussels & PO9 & 11/09/2019 & Brussels \\
\hline EEAS, Brussels & PO10 & $11 / 09 / 2019$ & Brussels \\
\hline EEAS, Brussels & PO11 & $11 / 09 / 2019$ & Brussels \\
\hline EEAS, Brussels & PO12 & $19 / 09 / 2019$ & Brussels \\
\hline EEAS, Brussels & PO13 & $19 / 09 / 2019$ & Brussels \\
\hline EEAS, Brussels & PO14 & 23/09/2019 & Phone interview \\
\hline EEAS, Brussels & PO15 & 03/10/2019 & Phone interview \\
\hline EEAS, Brussels & PO16 & 09/10/2019 & Brussels \\
\hline EEAS, Brussels & PO17 & $11 / 10 / 2019$ & Brussels \\
\hline EEAS, Brussels & PO18 & 04/12/2019 & Phone interview \\
\hline EEAS, Brussels & PO19 & 05/11/2019 & London \\
\hline EEAS, Brussels & PO20 & $05 / 06 / 2020$ & Phone interview \\
\hline Expert interview & $\mathrm{PO} 21$ & $26 / 03 / 2015$ & London \\
\hline Expert interview & $\mathrm{PO} 22$ & 02/07/2015 & Oxford \\
\hline EEAS, EU Delegation & $\mathrm{PO} 23$ & $29 / 04 / 2015$ & Phone interview \\
\hline EEAS, EU Delegation & $\mathrm{PO} 24$ & $12 / 05 / 2015$ & Phone interview \\
\hline EEAS, EU Delegation & PO25 & $15 / 05 / 2015$ & Phone Interview \\
\hline $\begin{array}{l}\text { European Commission, } \\
\text { DG NEAR }\end{array}$ & PO26 & $19 / 05 / 2015$ & Brussels \\
\hline EEAS, EU Delegation & $\mathrm{PO} 27$ & $27 / 05 / 2015$ & Phone interview \\
\hline EEAS, EU Delegation & PO28 & $27 / 05 / 2015$ & Phone interview \\
\hline EEAS, Brussels & PO29 & $29 / 05 / 2015$ & Phone interview \\
\hline
\end{tabular}




\begin{tabular}{|c|c|c|c|}
\hline Institution & $\begin{array}{l}\text { Transcript } \\
\text { code }\end{array}$ & Date of interview & $\begin{array}{l}\text { Location of } \\
\text { interview }\end{array}$ \\
\hline EEAS, EU Delegation & PO30 & $03 / 06 / 2015$ & Phone interview \\
\hline European Council & PO31 & $03 / 06 / 2015$ & Phone interview \\
\hline EEAS, OSCE & PO32 & $18 / 06 / 2015$ & Phone interview \\
\hline $\begin{array}{l}\text { Kati Piri, Member of the } \\
\text { European Parliament }\end{array}$ & PO33 & $23 / 06 / 2015$ & Brussels \\
\hline $\begin{array}{l}\text { European Commission, } \\
\text { DG NEAR }\end{array}$ & PO34 & $24 / 06 / 2015$ & Brussels \\
\hline EEAS, Brussels & PO35 & $10 / 07 / 2015$ & Phone interview \\
\hline EEAS, Brussels & PO36 & $28 / 07 / 2015$ & Brussels \\
\hline EEAS, EU Delegation & PO37 & $17 / 11 / 2015$ & Phone interview \\
\hline Seconded official & PO38 & $17 / 11 / 2015$ & Phone interview \\
\hline EEAS, Brussels & PO39 & $01 / 12 / 2015$ & Brussels \\
\hline EEAS, Brussels & PO40 & $01 / 12 / 2015$ & Brussels \\
\hline EEAS, Brussels & PO41 & $26 / 11 / 2015$ & Phone interview \\
\hline EEAS, Brussels & PO42 & $01 / 02 / 2016$ & Phone interview \\
\hline $\begin{array}{l}\text { Russian Mission to the } \\
\text { European Union }\end{array}$ & PO43 & 23/06/2016 & Brussels \\
\hline EEAS & PO44 & 07/03/2016 & Phone interview \\
\hline East StratCom Task Force & PO45 & $18 / 05 / 2016$ & Phone interview \\
\hline East StratCom Task Force & PO46 & $20 / 05 / 2016$ & Phone interview \\
\hline Seconded official & PO47 & $01 / 12 / 2015$ & Brussels \\
\hline EEAS, Brussels & PO48 & $22 / 06 / 2017$ & Phone interview \\
\hline EEAS, Brussels & PO49 & $23 / 06 / 2017$ & Phone Interview \\
\hline European Commission & PO50 & $29 / 06 / 2017$ & Phone interview \\
\hline EEAS, Brussels & PO51 & 03/07/2017 & Phone interview \\
\hline European Commission & PO52 & $04 / 07 / 2017$ & Phone interview \\
\hline EEAS, Brussels & PO53 & $11 / 07 / 2017$ & Phone interview \\
\hline East StratCom & PO54 & $10 / 07 / 2020$ & Phone interview \\
\hline European Defence Agency & PO55 & $13 / 07 / 2020$ & Phone interview \\
\hline European Defence Agency & PO56 & $20 / 07 / 2020$ & Phone interview \\
\hline EEAS, Brussels & PO57 & $20 / 07 / 2020$ & Phone interview \\
\hline DG Home & PO58 & $13 / 08 / 2020$ & Video interview \\
\hline East StratCom Task Force & PO59 & $14 / 08 / 2020$ & Phone interview \\
\hline DG Home & PO60 & $20 / 08 / 2020$ & Video interview \\
\hline East StratCom Task Force & PO61 & $24 / 08 / 2020$ & Video interview \\
\hline DG Home & PO62 & $27 / 08 / 2020$ & Video interview \\
\hline EEAS, Brussels & PO63 & $27 / 08 / 2020$ & Video interview \\
\hline DG Home & PO64 & $04 / 09 / 2020$ & Phone interview \\
\hline
\end{tabular}

